\title{
How flexibility affects the wake symmetry properties of a self-propelled plunging foil
}

\author{
Xiaojue Zhu, Guowei He and Xing Zhang † \\ The State Key Laboratory of Nonlinear Mechanics (LNM), Institute of Mechanics, \\ Chinese Academy of Sciences, Beijing 100190, China
}

(Received 11 July 2013; revised 26 May 2014; accepted 28 May 2014)

The wake symmetry properties of a flapping-foil system are closely associated with its propulsive performance. In the present work, the effect of the foil flexibility on the wake symmetry properties of a self-propelled plunging foil is studied numerically. We compare the wakes of a flexible foil and a rigid foil at a low flapping Reynolds number of 200. The two foils are of the same dimensions, flapping frequency, leading-edge amplitude and cruising velocity but different bending rigidities. The results indicate that flexibility can either inhibit or trigger the symmetry breaking of the wake. We find that there exists a threshold value of vortex circulation above which symmetry breaking occurs. The modification of vortex circulation is found to be the pivotal factor in the influence of the foil flexibility on the wake symmetry properties. An increase in flexibility can result in a reduction in the vorticity production at the leading edge because of the decrease in the effective angle of attack, but it also enhances vorticity production at the trailing edge because of the increase in the trailing-edge flapping velocity. The competition between these two opposing effects eventually determines the strength of vortex circulation, which, in turn, governs the wake symmetry properties. Further investigation indicates that the former effect is related to the streamlined shape of the deformed foil while the latter effect is associated with structural resonance. The results of this work provide new insights into the functional role of passive flexibility in flapping-based biolocomotion.

Key words: flow-structure interactions, propulsion, vortex streets

\section{Introduction}

Flapping wings/fins are used by birds, insects and fish to generate propulsive forces for locomotion. These appendages often undergo large deformation (passive or active) during flapping. A vast body of work concerning the functional role of passive flexibility in flapping wing systems can be found in the literature (see Shyy et al. (2010) for a comprehensive review). The effects of passive flexibility on performance enhancement and some physical mechanisms underlying these effects have been addressed in several studies (Katz \& Weihs 1978; Prempraneech, Hover \& Triantafyllou 2003; Heathcote \& Gursul 2007a; Michelin \& Smith 2009; Eldredge, Toomey \& Medina 2010; Spagnolie et al. 2010; Thiria \& Godoy-Diana

$\dagger$ Email address for correspondence: zhangx@lnm.imech.ac.cn 
2010; Ramananarivo, Godoy-Diana \& Thiria 2011; Kang et al. 2011; Shoele \& Zhu 2012).

In flapping-based animal locomotion, the wake symmetry properties are closely associated with the propulsive performance. First, a deflected wake (as the outcome of symmetry breaking) is always associated with a net lift and torque. Thus, animals may either exploit symmetry breaking when manoeuvring or avoid it when cruising. Second, for a deflected wake, the distribution of kinetic energy in the direction perpendicular to that of cruising can result in a reduction of propulsive efficiency. For rigid flapping-foil systems, the symmetry breaking of the reversed Kármán vortex street is a phenomenon that has been widely reported in the literature (Jones, Dohring \& Platzer 1998; Heathcote \& Gursul 2007b; Godoy-Diana, Aider \& Wesfreid 2008; Godoy-Diana et al. 2009; Cleaver, Wang \& Gursul 2012; Zheng \& Wei 2012). Despite the abundant literature concerning the effects of flexibility, studies of the relation between flexibility and wake symmetry properties are few. In a more recent study by Marais et al. (2012), it was reported that passive flexibility can inhibit wake symmetry breaking. In that experiment, the wake behind a flexible pitching foil was compared with that behind a rigid pitching foil. The two foils were of the same geometry and dimensions but composed of different materials. It was found that when both the flapping amplitude and chord-based Strouhal number were sufficiently large, although a deflected propulsive jet was observed for the rigid foil under these conditions, the jet remained symmetric in the case of the flexible foil. The stabilising effect of flexibility (in the sense of wake symmetry preservation) was explained by the fact that the deformation of the foil prevented the newly generated vortex at the trailing-edge from being sufficiently close to the previously shed vortex to form a vortex dipole. As symmetry breaking in a wake depends on the self-induced velocity of the dipole (Godoy-Diana et al. 2009), adding flexibility to the flapping foil thus inhibits the symmetry breaking of the wake.

The novel finding reported by Marais et al. (2012) has provided some new insights into the role of passive flexibility in propulsion. This finding is also beneficial to the design of efficient artificial underwater vehicles. However, because of the limitations of the experimental method, only a narrow range of parameter space (especially in bending rigidity) was investigated by Marais et al. (2012). To fully understand how flexibility affects the vortex dynamics governing the symmetry breaking of the wake, a parametric sweep over a much wider range is necessary. In this regard, computational approaches are more cost-effective than experimental ones. Motivated by the observations of Marais et al. (2012) and the desire to better understand the role of passive flexibility in biolocomotion, we perform a fluid-structure interaction (FSI) simulation of the self-propulsion of a flexible plunging foil. To the best of the authors' knowledge, this is the first numerical investigation of the effect of flexibility on wake symmetry properties. By probing a much larger parameter space, we demonstrate that flexibility can either inhibit or trigger wake symmetry breaking. The modification of vortex circulation is found to be the pivotal factor in the influence of flexibility on wake symmetry properties. An in-depth analysis indicates that the modification of vortex circulation can be further correlated with the change in the effective angle of attack and the change in the trailing-edge flapping velocity of the foil. In addition, the importance of the effects associated with structural resonance in defining the wake pattern (symmetric or asymmetric) is also studied.

The reminder of the paper is organised as follows. In $\S 2$, we describe the model and the immersed boundary method for the FSI simulations. Section 3 contains four subsections. First, based on the simulations performed, we present the observational 


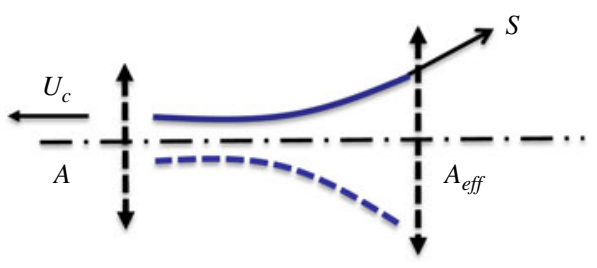

FIgURE 1. (Colour online) Schematic depiction of the model.

wake patterns in the parameter space. Then, a vortex dipole model is introduced to characterise the wake symmetry properties. In the third subsection, the underlying principle and mechanism of wake transition are discussed. In the fourth subsection, the relation between structural resonance and wake symmetry properties is addressed. Finally, some conclusions are drawn in $\S 4$.

\section{Computational model and methodology}

Two different models exist for the hydrodynamic study of flapping-foil systems. The first model is a tethered flapping foil immersed in a uniform oncoming flow (or, equivalently, a flapping foil towed at a given speed through a fluid). The second model is a flapping foil under the self-propelled (or free-swimming) condition. Here, we underline the essential difference between these two models: in the former model, the forward speed and flapping motion are decoupled and thus can be independently prescribed, whereas in the latter, the forward speed is the result of the complex FSI problem and cannot be prescribed. The former model has been used in most previous studies because of its simplicity. It is only recently that the latter model has been widely adopted in research efforts (Spagnolie et al. 2010; Thiria \& Godoy-Diana 2010; Zhang, Liu \& Lu 2010; Ramananarivo et al. 2011; Alben et al. 2012; Lee \& Lee 2013). Because the self-propelled model can better represent real-world biological and biomimetic locomotion (Lauder et al. 2007), we adopt this model for the present study.

A schematic representation of the model is presented in figure 1. The flapping foil is modelled as an inextensible flexible filament that is driven at the leading edge by a harmonically plunging motion. In the horizontal direction, the filament is unconstrained. The flow is assumed to be two-dimensional, incompressible and laminar.

The motions of the fluid and the filament are governed by the Navier-Stokes equations coupled with a geometrically nonlinear structural equation (Huang, Shin \& Sung 2007):

$$
\begin{gathered}
\frac{\partial \boldsymbol{u}}{\partial t}+(\boldsymbol{u} \cdot \nabla) \boldsymbol{u}=-\nabla \boldsymbol{p}+\frac{1}{R e_{f}} \nabla^{2} \boldsymbol{u}+\boldsymbol{f}, \\
\beta \frac{\partial^{2} \boldsymbol{X}}{\partial t^{2}}-\frac{\partial}{\partial s}\left(\zeta(s) \frac{\partial \boldsymbol{X}}{\partial s}\right)+\frac{\partial^{2}}{\partial s^{2}}\left(\gamma \frac{\partial^{2} \boldsymbol{X}}{\partial s^{2}}\right)=-\boldsymbol{F}, \\
\frac{\partial \boldsymbol{X}}{\partial s} \cdot \frac{\partial \boldsymbol{X}}{\partial s}=1,
\end{gathered}
$$

where $\boldsymbol{u}, p$ and $\boldsymbol{X}=\left(X^{(1)}, X^{(2)}\right)$ are the fluid velocity, fluid pressure and displacement of the filament, respectively, and $\boldsymbol{f}$ and $\boldsymbol{F}$ are the Eulerian and Lagrangian force 
densities, respectively, that represent the interaction between the fluid and the structure. The nonlinearity of the structural equation stems from the tension $\zeta$ that enforces the inextensibility condition $\partial \boldsymbol{X} / \partial s \cdot \partial \boldsymbol{X} / \partial s=1$. Here $\zeta$ is a function of the Lagrangian coordinate $s$ and is governed by a Poisson equation, which can be derived using the equation of structural displacement and the inextensibility condition (Huang et al. 2007).

Equation (2.1) is written in a dimensionless form, which is obtained by scaling space and time with the filament length $L$ and with $L / U_{\text {ref }}$, respectively $\left(U_{\text {ref }}=\pi A f\right.$ is the maximum flapping velocity, where $A$ and $f$ are the peak-to-peak flapping amplitude and the flapping frequency, respectively). The dimensionless numbers that appear in (2.1) are as follows: $R e_{f}=\left(\pi \rho_{f} A f L\right) / \mu, \gamma=B /\left(\rho_{f} U_{r e f}^{2} L^{3}\right), \zeta(s)=T(s) /\left(\rho_{f} U_{r e f}^{2} L\right)$, $\beta=\rho_{s} /\left(\rho_{f} L\right)$ and $\bar{A}=A / L$, which are the flapping Reynolds number, the dimensionless bending rigidity, the dimensionless tension, the mass ratio and the relative amplitude, respectively. Here $\rho_{f}$ is the area density of the fluid, $\rho_{s}$ is the linear density of the filament and $\mu$ is the dynamic viscosity of the fluid. Here $B$ and $T$ are the dimensional bending rigidity and tension, respectively. The dimensionless form of the prescribed plunging motion at the leading edge is found to be $y(t)=(\bar{A} / 2) \cos [(2 / \bar{A}) \cdot t]$ by following the same scaling procedure.

For the structure, the boundary condition that combines the vertically forced oscillation, the clamped condition and the horizontally unconstrained condition, i.e. $X^{(2)}=y(t), \partial \boldsymbol{X} / \partial s=(1,0)^{\mathrm{T}}, \partial^{3} X^{(1)} / \partial s^{3}=0$ is enforced at $s=0$ (the leading edge). A free-end condition, i.e. $\partial^{2} \boldsymbol{X} / \partial s^{2}=(0,0)^{\mathrm{T}}, \partial^{3} \boldsymbol{X} / \partial s^{3}=(0,0)^{\mathrm{T}}$ is imposed at $s=1$ (the trailing edge). For the fluid, a no-slip condition is enforced at the outer boundaries. Here $\boldsymbol{X}(s, 0)=(s, \bar{A} / 2)^{\mathrm{T}}, \dot{\boldsymbol{X}}(s, 0)=(0,0)^{\mathrm{T}}$ is the initial condition for the structure and $\boldsymbol{u}(\boldsymbol{x}, 0)=0$ is the initial condition for the fluid.

The simulations were performed using the immersed boundary method for the fluid flow and the finite-difference method for the motion of the structure. In the immersed boundary method, the motions of the fluid and the structure are coupled at their interface by the no-slip condition $\int_{V} \boldsymbol{u}(\boldsymbol{x}, t) \delta(\boldsymbol{x}-\boldsymbol{X}) \mathrm{d} \boldsymbol{x}=\dot{\boldsymbol{X}}$ and by the relation between the Lagrangian and Eulerian forces, $\boldsymbol{f}(\boldsymbol{x}, t)=\int_{s} \boldsymbol{F}(\boldsymbol{X}(s), t) \delta(\boldsymbol{x}-\boldsymbol{X}(s)) \mathrm{d} s$, where $\delta$ is the discrete delta function. Extensive validations of the immersed boundary solver have been performed by Wang \& Zhang (2011) (for flows past rigid objects) and Zhu, He \& Zhang (2014) (for FSI problems).

A large rectangular computational domain of $58 \mathrm{~L} \times 12 \mathrm{~L}$ in size is used in the simulations performed in this study, with a grid width of $0.02 L$. The time steps are chosen such that the CFL number is fixed to 0.5. Grid-convergence tests were conducted to ensure that the results obtained are independent of the mesh resolution. Figure 2 presents the swimming velocity and the resultant horizontal and vertical forces as functions of time for three different grid widths (the other control parameters in the tests are as follows: $\operatorname{Re}_{f}=200, \bar{A}=1.0, \beta=0.2$ and $\gamma=4.0$ ). For the swimming velocity, some deviation among the results can be observed when the mesh width is halved from $0.04 L$ to $0.02 L$. However, when the grid width is further halved (from $0.02 L$ to $0.01 L$ ), no significant additional change in the result is observed. For the horizontal force, a similar trend of mesh convergence is apparent from the figure. For the vertical force, the results obtained using all of the three meshes are nearly indistinguishable.

Some important physical quantities used to characterise the propulsive performance and vortex shedding are defined as follows. The effective amplitude $A_{\text {eff }}$ is defined as the peak-to-peak amplitude measured at the trailing edge, and the amplitude ratio is 

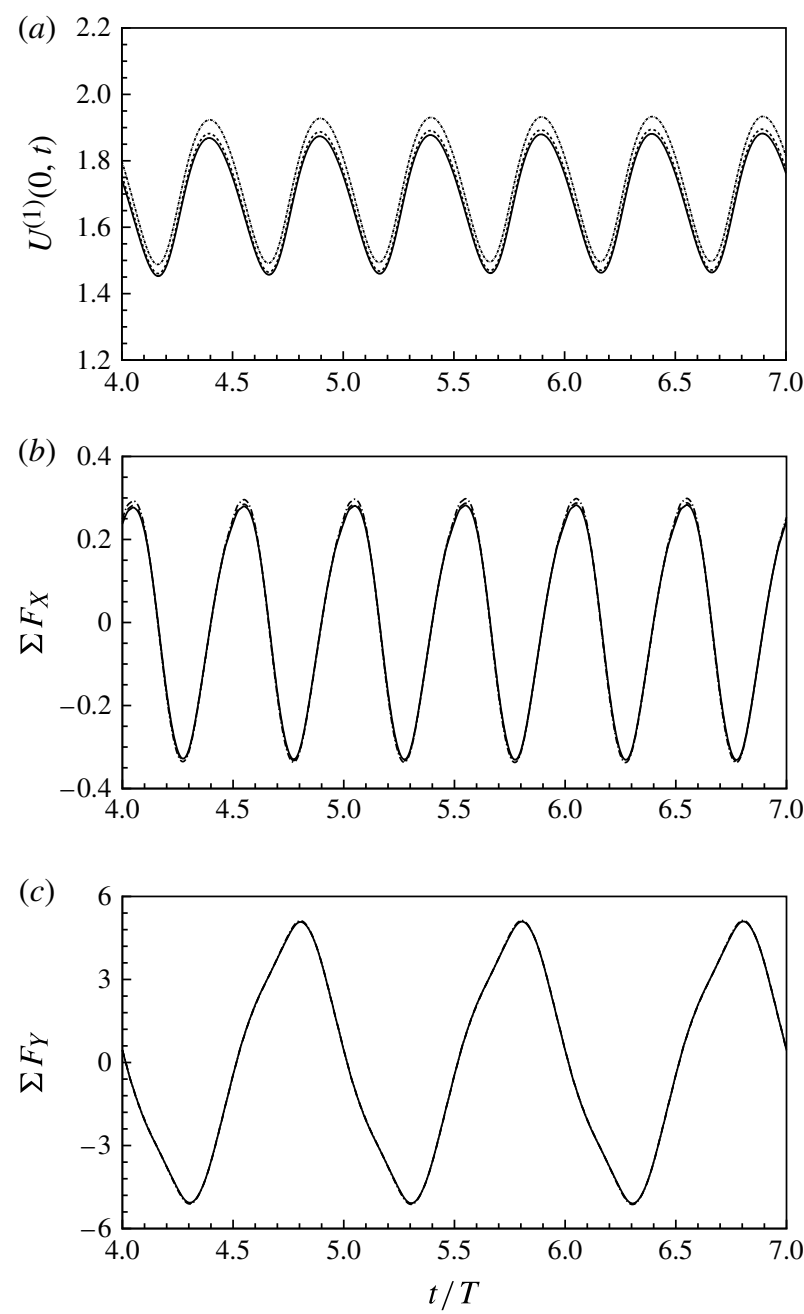

FIGURE 2. Tests of mesh independence: the time histories of $(a)$ the swimming velocity $\left(U^{(1)}(0, t)=\partial_{t} X^{(1)}(0, t)\right)$ and the resultant $(b)$ horizontal and $(c)$ vertical forces for grid widths of $0.04 \mathrm{~L}$ (dash-dotted line), $0.02 \mathrm{~L}$ (dashed line) and $0.01 \mathrm{~L}$ (solid line). Here $T=\pi \bar{A}$ is the dimensionless oscillation period. An average horizontal force of zero is obtained by integrating the curve in $(b)$, thus confirming that a periodically steady state has been achieved.

defined as $A_{\text {eff }} / A$. The (dimensionless) cruising velocity $U_{c}$ is defined as the average horizontal velocity evaluated at the leading edge when periodicity is reached,

$$
U_{c}=1 /(\pi \bar{A}) \int_{0}^{\pi \bar{A}}\left|\partial_{t} X^{(1)}(0, t)\right| \mathrm{d} t .
$$

The Strouhal number based on the filament length is defined as

$$
S t_{L}=(f L) / \tilde{U}_{c}=1 /\left(\pi \bar{A} U_{c}\right),
$$

where $\tilde{U}_{c}$ is the dimensional cruising velocity. Similarly, the Strouhal number based on the trailing-edge amplitude is defined as 


$$
S t_{A}=\left(f A_{e f f}\right) / \tilde{U}_{c}=S t_{L} \cdot \bar{A} \cdot\left(A_{e f f} / A\right) .
$$

It should be noted that for a self-propelled foil, $A_{e f f} / A, S t_{L}$ and $S t_{A}$ are also the results of the FSI problem and cannot be prescribed.

The input power required to produce the oscillation of the foil can be defined as

$$
P_{s}=\frac{1}{\pi \bar{A}} \int_{0}^{\pi \bar{A}}\left(\int_{0}^{1}\left(\boldsymbol{F} \cdot \frac{\partial \boldsymbol{X}}{\partial t}\right) \mathrm{d} s\right) \mathrm{d} t .
$$

Further explanation is needed regarding the definition of propulsive efficiency in a self-propelled (free-swimming) foil. For a tethered flapping foil immersed in an oncoming flow, the propulsive efficiency is usually defined as the ratio of the useful power over the input power, where the useful power can be simply calculated as the average thrust force times the forward velocity (Anderson et al. 1998). However, in a self-propelled foil, this definition is not appropriate for quantifying the propulsive efficiency (Schultz \& Webb 2002) because the foil experiences a zero average net thrust in the periodically steady state (see figure $2 b$ ). Under such circumstances, no consensus has been reached among researchers concerning the correct way to quantify the propulsive efficiency. At present, three different definitions of this 'efficiency' can be found in the literature. Some researchers continue to use the definition for a tethered foil and attempt to isolate the 'thrust' from the horizontal force (Borazjani \& Sotiropoulos 2008). Because 'thrust' and 'drag' are inseparably related under the self-propulsion condition (Schultz \& Webb 2002), this type of force 'decomposition' is necessarily somewhat arbitrary. Alternatively, other researchers have measured the 'thrust' by conducting another independent experiment (in addition to the free-swimming one) with the flapping foil held in a fixed position (Thiria \& Godoy-Diana 2010; Ramananarivo et al. 2011). The third type of 'efficiency' used by some researchers is the ratio of the 'kinetic energy' that is eventually gained in the forward motion over the average 'input work' during one period of flapping (Kern \& Koumoutsakos 2006; Zhang et al. 2010). We prefer to use this last definition because of the clear physical meaning it conveys. In fact, this definition of efficiency for a self-propelled flapping foil is closely associated with the conventional definition for a tethered flapping foil, in the sense that the 'kinetic energy' that is eventually gained in a self-propelled foil can be related to the amount of 'useful power' delivered during the initial period before the periodically steady state is achieved. As a mathematical formula, this type of efficiency is defined as

$$
\eta=\frac{\frac{1}{2} \beta U_{c}^{2}}{\pi \bar{A} P_{s}} .
$$

\section{Results and discussion}

There are four control parameters in this problem: $R e_{f}, \bar{A}, \beta$ and $\gamma$. The values of the control parameters used in the simulations are listed in table 1 . The Reynolds number used in the simulations is 200, which is comparable with the values used in some previous works, such as Godoy-Diana et al. (2008) and Marais et al. (2012).

\subsection{Wake patterns in parameter space}

Figure 3 summarises the observed wake patterns in the parameter space. The wake patterns in the two-dimensional parameter space $\left(A_{\text {eff }} / A, S t_{L}\right)$ are presented for $\bar{A}=1.6$ 


$\begin{array}{cccccc}\text { Series } & R e_{f} & \bar{A} & \beta & \gamma & \text { Symbol } \\ 1 & 200 & 1.6 & 0.4 & 10^{-1}-10^{4} & \ominus \\ 2 & 200 & 1.6 & 0.8 & 10^{-1}-10^{4} & \otimes \\ 3 & 200 & 1.6 & 1.6 & 10^{-1}-10^{4} & \oplus \\ 4 & 200 & 1.6 & 2.0 & 10^{-1}-10^{4} & \oslash \\ 5 & 200 & 1.0 & 0.1 & 10^{-3}-10^{4} & \boldsymbol{\nabla} \\ 6 & 200 & 1.0 & 0.2 & 10^{-3}-10^{4} & \boldsymbol{\Delta} \\ 7 & 200 & 1.0 & 0.7 & 10^{-1}-10^{4} & \bullet \\ 8 & 200 & 1.0 & 1.0 & 10^{-1}-10^{4} & \bullet \\ 9 & 200 & 1.0 & 2.0 & 10^{-1}-10^{4} & \bullet \\ 10 & 200 & 0.4 & 0.2 & 10^{-3}-10^{4} & + \\ 11 & 200 & 0.4 & 0.6 & 10^{-1}-10^{4} & * \\ 12 & 200 & 0.4 & 2.0 & 10^{-1}-10^{4} & \boldsymbol{\square} \\ 13 & 200 & 0.5-0.9 & 0.2 & 10^{4} & \bullet\end{array}$

TABLE 1. Values of the control parameters used in the simulations.

(series 1-4 in table 1), $\bar{A}=1.0$ (series 5-9 in table 1) and $\bar{A}=0.4$ (series 10-12 in table 1) in figures $3(a), 3(b)$, and $3(c)$, respectively. For $\bar{A}=1.6$ and $\bar{A}=1.0$, the symmetric and asymmetric wake regions are separated by both a lower border and an upper border, whereas for $\bar{A}=0.4$, there is only one wake-transition border. In all scenarios, the amplitude ratio is found to be crucial in dictating the wake symmetry properties. The wake patterns in the three-dimensional parameter space $\left(A_{\text {eff }} / A, \bar{A}, S t_{L}\right)$ are presented in figure $3(d)$. Figure $3(a-c)$ can be regarded as three parallel slices of this diagram with constant $\bar{A}$. It is apparent that the symmetric wake region (region I) lies between the two borders $s_{1}$ and $s_{2}$, whereas the two asymmetric wake regions lie below $s_{1}$ (region II) or above $s_{2}$ (region III). This diagram is a natural extension of the $\left(\bar{A}, S t_{L}\right)$ map that is used for the study of wake transitions in a rigid flappingfoil system (Godoy-Diana et al. 2008). The transition from a symmetric wake to an asymmetric wake for a rigid flapping foil occurs by crossing curve $c_{1}$ on the plane of $A_{\text {eff }} / A=1.0$ in figure $3(d)$. For a flexible foil, the transition from region II (asymmetric wake) to region I (symmetric wake) by crossing surface $s_{1}$ corresponds to the finding presented by Marais et al. (2012) that, flexibility inhibits symmetry breaking. In this study, we have discovered a new asymmetric wake region (region III). The transition from region I to region III by crossing surface $s_{2}$ corresponds to the novel finding that flexibility can also trigger symmetry breaking. Note that the separation borders depicted in figure 3 were crudely estimated based on the available simulated cases. As the purpose of plotting these diagrams is to qualitatively explain the two opposing effects of flexibility on wake symmetry properties, no further effort has been made to determine the precise locations of the transitional borders.

\subsection{A vortex dipole model for characterising wake symmetry properties}

To demonstrate that adding flexibility can either inhibit or trigger symmetry breaking, we consider foils of the same Reynolds number, relative amplitude and mass ratio but different bending rigidities (one flexible and the other rigid) and examine the symmetry properties of their wakes. Two pairs of such analogous cases are labelled as (A1, A2) and (B1, B2) in figures $3(b)$ and 3(c), respectively. The values of certain important parameters for these four cases are listed in table 2. It is evident that for 

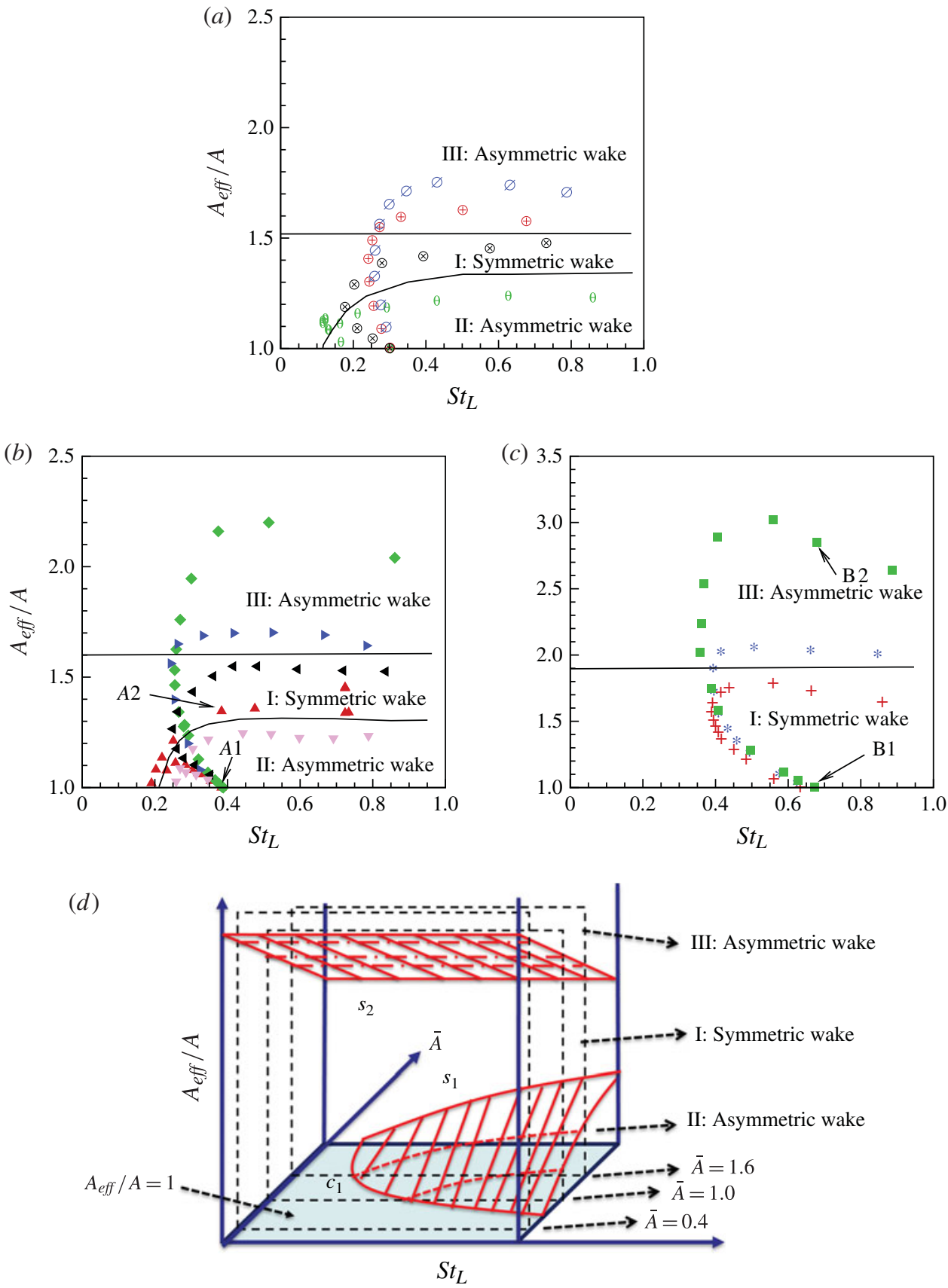

FIGURE 3. (Colour online) Wake patterns in parameter space: (a) two-dimensional parameter space at $\bar{A}=1.6 ;(b)$ two-dimensional parameter space at $\bar{A}=1.0 ;$ (c) two-dimensional parameter space at $\bar{A}=0.4$; and $(d)$ three-dimensional parameter space. The symbols used to present the data in $(a-c)$ are defined in table 1 .

each pair of cases, the Strouhal numbers $S t_{L}$ achieved by the rigid and flexible foils are the same. The two opposing effects of flexibility on symmetry breaking can be 


$\begin{array}{ccccccc} & \bar{A} & \beta & \gamma & U_{c} & S t_{L} & \eta \\ \mathrm{A} 1 & 1.0 & 0.2 & 10^{4} & 0.83 & 0.38 & 0.11 \\ \mathrm{~A} 2 & 1.0 & 0.2 & 0.1 & 0.83 & 0.38 & 0.31 \\ \mathrm{~B} 1 & 0.4 & 2.0 & 10^{4} & 1.17 & 0.68 & 0.33 \\ \mathrm{~B} 2 & 0.4 & 2.0 & 3.6 & 1.17 & 0.68 & 0.11\end{array}$

TABLE 2. Values of selected physical quantities in cases A1, A2, B1 and B2.

(a)

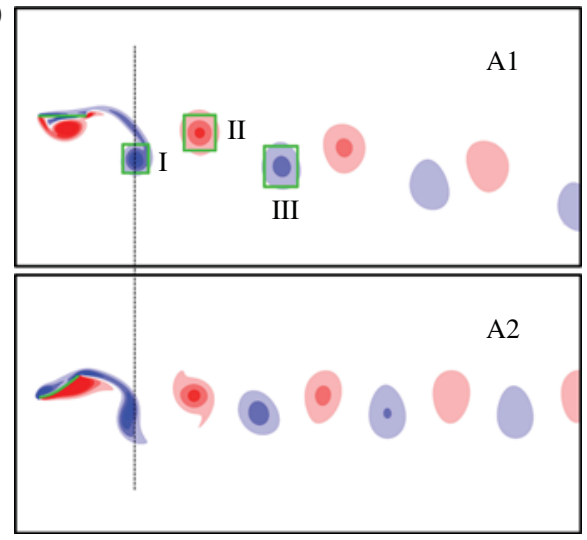

(b)

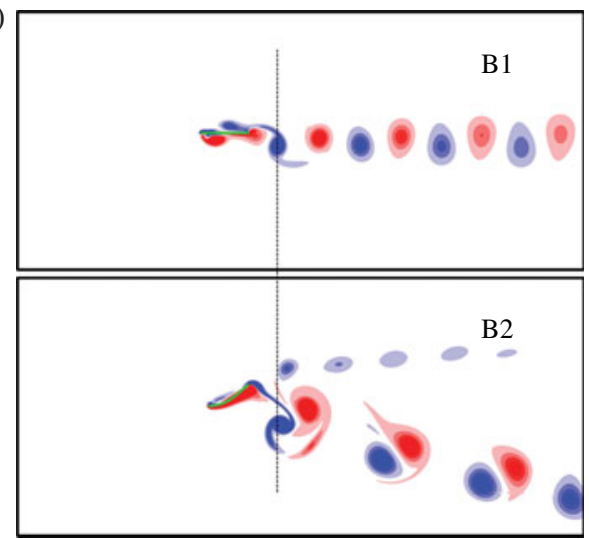

FIgURE 4. (Colour online) Vorticity contours in the wakes for (a) pair (A1, A2) and (b) pair (B1, B2).

clearly observed from the wake structures depicted in figure 4 . When the flexibility of the foil is increased, wake symmetry breaking is inhibited in pair (A1, A2) but triggered in pair (B1, B2). Another notable observation is that for the cases with asymmetric wakes, the propulsive efficiency is much lower compared with the cases with symmetric wakes (see table 2).

In the two asymmetric cases (A1 and B2), both wakes deflect downwards. After further investigation, we find that the preferred direction of wake deflection depends on the initial conditions. When the initial position of the filament is set to $\boldsymbol{X}(s, 0)=(s,-\bar{A} / 2)^{\mathrm{T}}$, upward deflection is observed in both cases. Another interesting finding is that although the tilting direction of the wake depends on the initial conditions, the wake symmetry properties are not similarly affected. To confirm this, we carefully examine two quantitative indicators of the wake-deflection trend, namely, the symmetry-breaking effective phase velocity and the symmetry-preserving effective phase velocity (their definitions are provided below), and we find that the magnitudes of these two velocities remain the same under various initial conditions.

In the vortex dipole model proposed by Godoy-Diana et al. (2009), the effective phase velocity of a dipole is used to quantify the trend of wake deflection. The definition of the effective phase velocity is

$$
U_{p}^{*}=U_{\text {dipole }}-U_{\text {phase }} \cos \alpha,
$$

where $U_{\text {phase }}$ is the average streamwise velocity of the two vortex centres of a dipole (the centre of a vortex is defined as the location of maximum vorticity), $\alpha$ is the orientation angle between the streamwise direction and the direction of the dipole (see 


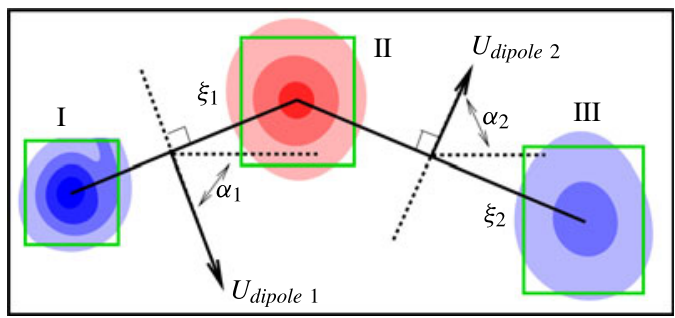

FIGURE 5. (Colour online) A schematic illustration of two consecutive dipoles in the very near wake.

figure 5), and $U_{\text {dipole }}$ is the velocity induced by a dipole. This last quantity can be defined as

$$
U_{\text {dipole }}=\Gamma /(2 \pi \xi),
$$

where $\Gamma$ is the circulation of the dipole and $\xi$ is the distance between the two vortex centres of the dipole. Here $\Gamma$ is calculated by integrating the vorticity over a rectangular area, as shown in figure 5. The size of the integration area is determined by performing Gaussian fits, $\mathrm{e}^{\left(-x_{i}^{2} / \sigma_{i}^{2}\right)}$, along the vertical and horizontal axes centred on the positions of maximum vorticity. The sizes of the vortex along the $x$ and $y$ directions are then defined as $2 \sigma_{i}$. The circulation that appears in (3.2) is the average value for the two vortices in the dipole.

A symmetry-breaking condition $U_{p}^{*}<0$ was first proposed by Godoy-Diana et al. (2009). Another condition involving two effective phase velocities of the two consecutive dipoles (see figure 5) was later proposed by Zheng \& Wei (2012). Wake symmetry is preserved if the two effective phase velocities are identical; otherwise, symmetry breaking occurs. In all asymmetric cases in this study, the wakes are deflected downwards. Thus, the effective phase velocity of the dipole formed by vortices I and II in figure 5 is called the symmetry-breaking effective phase velocity. Similarly, the effective phase velocity of the dipole formed by vortices II and III is called the symmetry-preserving effective phase velocity.

The time histories of the $x$ and $y$ positions of the three vortices in the four cases are presented in figure 6 . Here $T=\pi \bar{A}$ is the dimensionless flapping period. Tracking begins at the moment when the direction of motion of the trailing edge reverses (corresponding to the birth of a new vortex). In all cases, the three vortices move with constant velocity in the streamwise direction. The streamwise velocity in case A2 is smaller than that in case A1, whereas the streamwise velocity in case B2 is larger than that in case B1. The vertical velocities in cases A2 and B1 are found to be zero, whereas negative vertical velocities are observed in cases A1 and B2. It is also apparent that the magnitude of the vertical velocity in case B2 is much larger than that in case A1. This finding is consistent with the wake structures presented in figure 4.

For each case, the phase velocity $U_{\text {phase }}$ is computed from the time history of the $x$ position. The physical quantities of the dipoles in each of the four cases are summarised in table 3 . The quantities presented in table 3 are good indicators of the wake symmetry properties and can aid in obtaining a better understanding of the wake dynamics. It is evident that for cases A2 and B1, the two effective phase velocities are identical. For cases A1 and B2, however, the symmetry-breaking effective phase velocity is larger than the symmetry-preserving effective phase velocity. The difference in these two velocities can be primarily attributed to the fact that the 
(a)

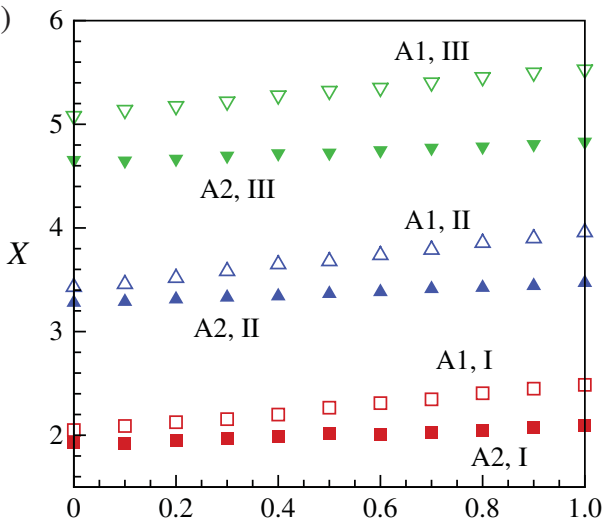

(c)

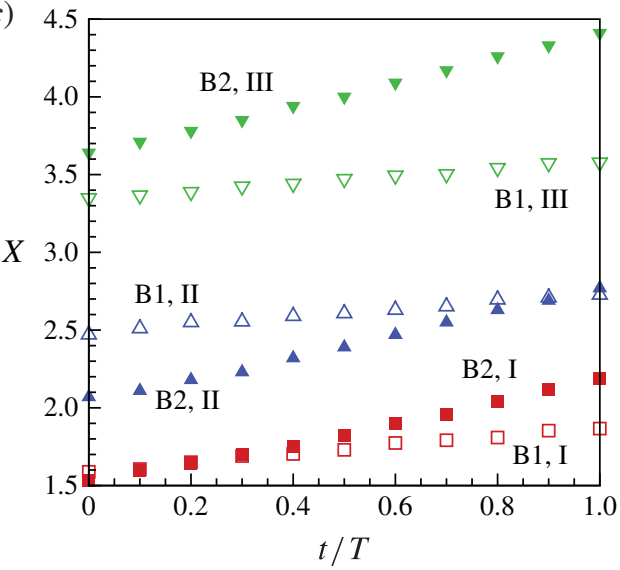

(b)

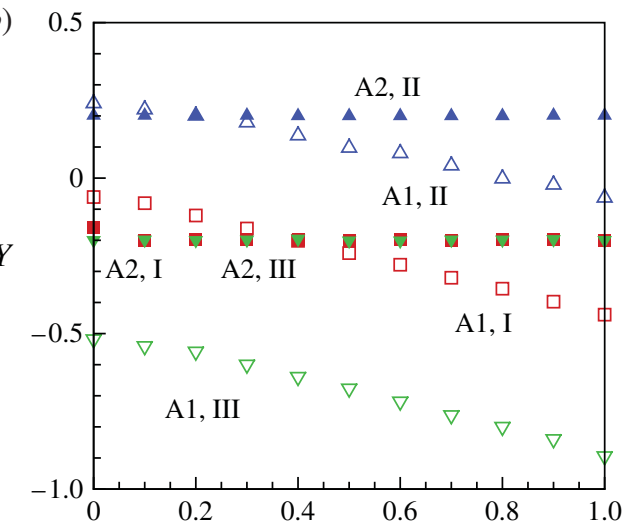

(d)

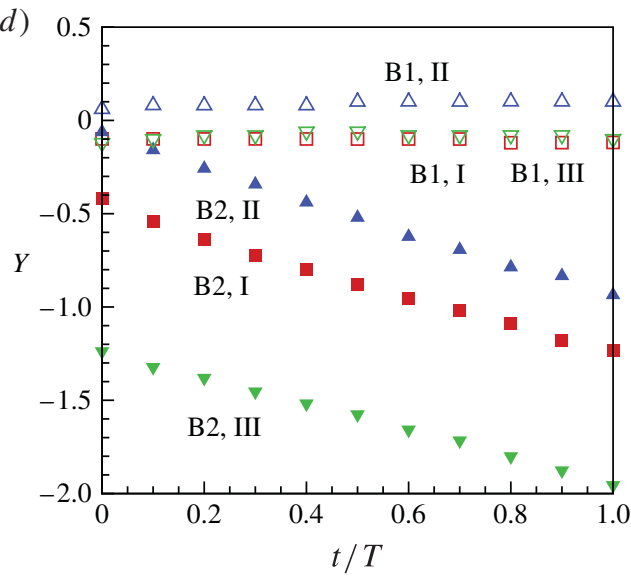

FIguRE 6. (Colour online) The time histories of the $x$ and $y$ positions of vortices: $(a) x$ positions in $\mathrm{A} 1$ and $\mathrm{A} 2$; $(b) y$ positions in $\mathrm{A} 1$ and $\mathrm{A} 2$; $(c) x$ positions in $\mathrm{B} 1$ and $\mathrm{B} 2$; and (d) $y$ positions in B1 and B2. Here $T=\pi \bar{A}$ is the dimensionless oscillation period. Squares, triangles and inverted triangles denote the positions of vortex I, II and III, respectively. The empty symbols denote the positions for the rigid cases (A1 and B1), whereas the filled symbols denote the positions for the flexible cases (A2 and B2). The origins of the $x$ - and $y$-axes correspond to the leading edge of the foil and the equilibrium position, respectively.

distance $\xi_{1}$ in the symmetry-breaking dipole is smaller than the distance $\xi_{2}$ in the symmetry-preserving dipole. Thus, the validity of the criterion proposed by Zheng \& Wei (2012) is confirmed in all four cases. This finding also confirms the consistency between the data listed in table 3 and the wake patterns presented in figure 4, thereby increasing our confidence in the computational procedure used to obtain the data (despite the uncertainties in the Gaussian fits, vorticity truncation, etc.). In the present study, the criterion proposed by Zheng \& Wei (2012) is also used to aid in the identification of wake patterns.

\subsection{Underlying principle and mechanism of wake transition}

In a study by Marais et al. (2012), it was observed that because of the deformation of the pitching foil, vortices were shed at a position that was horizontally nearer to 


$\begin{array}{ccccccccc} & U_{p}^{* 1} & U_{p}^{* 2} & \Gamma & U_{\text {phase }} & \xi_{1} & \xi_{2} & \alpha_{1} \text { (deg.) } & \alpha_{2} \text { (deg.) } \\ \text { A1 } & 0.22 & 0.14 & 2.38 & 0.15 & 1.40 & 1.90 & 18.5 & 24.5 \\ \text { A2 } & 0.12 & 0.12 & 1.22 & 0.05 & 1.42 & 1.42 & 16.2 & 16.4 \\ \text { B1 } & 0.26 & 0.26 & 1.68 & 0.21 & 0.88 & 0.88 & 11.2 & 12.7 \\ \text { B2 } & 0.33 & 0.10 & 3.01 & 0.55 & 0.66 & 1.94 & 32.1 & 33.7\end{array}$

TABLE 3. The values of certain quantities for the two dipoles in the four considered cases. Here $U_{p}^{* 1}$ and $U_{p}^{* 2}$ denote the effective phase velocities of the dipoles formed by vortices (I, II) and vortices (II, III), respectively.

the pivot point in the flexible case than in the rigid case. The stabilising effect of flexibility was thus attributed to the alteration in the horizontal position of the newly formed vortices. In this study, the kinematics of the foil is very different from that in Marais et al. (2012), and the effects of flexibility on the symmetry breaking of the wake are also found to be different. From figures 4 and 6 , it is evident that the vortices are shed at nearly the same horizontal positions in both the rigid and flexible cases. Thus, we believe that in the present problem, the mechanism through which the flexibility affects the wake symmetry properties does rely on altering the positions of the newly formed vortices.

In the present study, we find that the circulation of vortices may play a crucial role in dictating the symmetry properties of wake. As shown in table 3, for pair (A1, A2), in which increased flexibility inhibits symmetry breaking, the vortex circulation is reduced by increasing the flexibility of the foil, whereas for pair (B1, B2), in which increased flexibility triggers symmetry breaking, the vortex circulation is enhanced by increasing the flexibility. This connection between vortex circulation and wake symmetry properties is intuitively reasonable, because if there is any minor difference in the geometric factors (such as $\xi$ ) of the two dipoles caused by small perturbation, then larger vortex circulation will lead to a greater difference between the two effective phase velocities (see (3.2)). Similar phenomena have also been reported in a number of recent studies. Liang et al. (2011) and Zheng \& Wei (2012) have found that stronger vortex circulation attributable to an increased Reynolds number tends to destabilise a wake (in the sense of symmetry preservation). Cleaver et al. (2012) have found a minimum value of dimensionless circulation to be necessary for symmetry breaking to occur, and they interpreted this number as the inverse of a modified Strouhal number based on the dipole velocity. From table 3 , it can be inferred that in the current study, this threshold value must lie somewhere between 1.68 and 2.38. To determine this value more precisely, we examine the circulation and wake type in numerous cases and find that the threshold value is approximately 2.0 (see the $\left(\Gamma, S t_{A}\right)$ map in figure $\left.7 a\right)$, which is very close to 1.85 , the value reported by Cleaver et al. (2012).

Several transition paths between symmetric and asymmetric wakes on the $\left(\Gamma, S t_{A}\right)$ map are illustrated in figure 7(b). The transition from a symmetric wake to an asymmetric wake (or vice versa) near intersection 'a' (by crossing $\Gamma=\Gamma^{*}$ and $S t_{A}=S t_{A}^{*}$ simultaneously with $S t_{A}^{*}$ close to 0.30 ) corresponds to previous observations of rigid flapping foils (Godoy-Diana et al. 2008). The transitions that occur near intersections ' $b$ ' (path 1) and ' $d$ ' (path 2) correspond to the finding that flexibility can inhibit symmetry breaking (Marais et al. 2012). The transitions that take place near 'c' (in path 1) and 'e' (in path 3) correspond to the novel finding of this study that flexibility can also trigger symmetry breaking. 
(a)

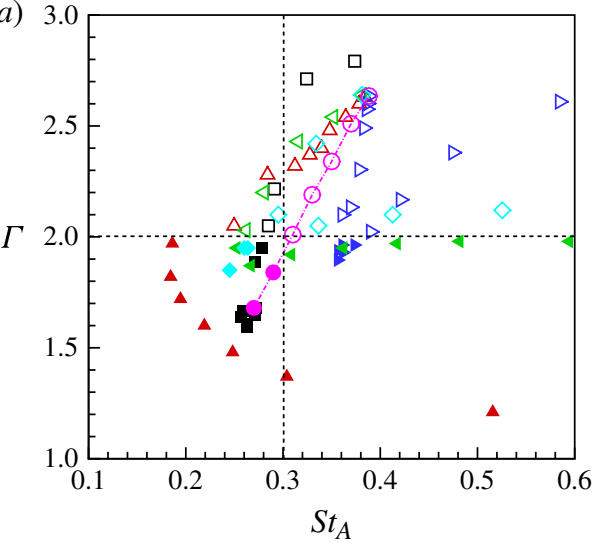

(b)

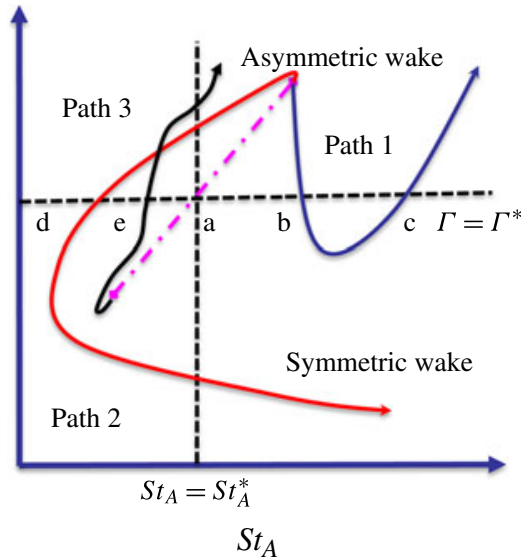

FIgURE 7. (Colour online) The plot of circulation versus Strouhal number and the wake-transition paths: (a) $\Gamma$ versus $S t_{A}$ plot and $(b)$ transition paths. The symbols used to present the data in $(a)$ are defined in table 1; filled and empty symbols represent symmetric and asymmetric wakes, respectively. The rigid cases are connected by a dash-dotted line in $(a)$ and $(b)$. The solid lines with arrows in $(b)$ denote the paths for flexible foils; each arrow represents the direction of decreasing $\gamma$.

We now examine the process of vortex formation procedure to elucidate the mechanism through which flexibility modulates vortex circulation. Figure 8 depicts the vortex structures in the near wake for the four considered cases at five different moments during one flapping cycle. It is apparent that in all four cases, all wakes are of the ' $2 S$ ' type (in which two vortices of opposite signs are shed per oscillation period). During each stroke, the vortex structure formed at the leading edge merges with the vortex generated at the trailing edge before the merged structure is shed into the wake as one single vortex. Thus, the circulation of the vortices that are shed into the wake depends on vorticity contribution from both the leading edge and the trailing edge. In figure 8, a large separation bubble near the leading edge can be clearly observed in the rigid cases (A1 and B1), whereas for the flexible cases (A2 and B2), the deformation of the foil suppresses the formation of a well-established leading-edge vortex (LEV) structure.

The primary factor affecting vorticity production at the leading edge is the effective angle of attack, which can be defined as

$$
\alpha_{\text {eff }}(t)=\phi-\theta=\arctan \left(\frac{U_{L 2}}{U_{L 1}}\right)-\theta .
$$

Here, $\phi$ is the angle of attack seen by a rigid foil and $\theta$ is the deflection angle associated with foil deformation (see figure 9). The time histories of the effective angles of attack are presented in figure 10. From this figure, it is evident that because of the deformation of the foil, the effective angle of attack is considerably reduced in the flexible cases (A2 and B2) compared with the rigid cases (A1 and B1). As suggested by Anderson et al. (1998) and Shoele \& Zhu (2012), a decrease in the effective angle of attack reduces the scale of separation and thus the vorticity generation at the leading edge.

The primary factor affecting vorticity production at the trailing edge is the trailingedge flapping velocity $U_{T 2}$ (see figure 9). The time histories of the trailing-edge 
(a)

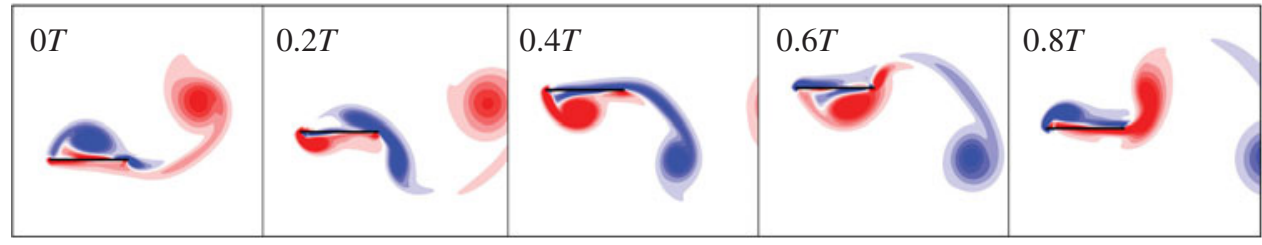

(b)

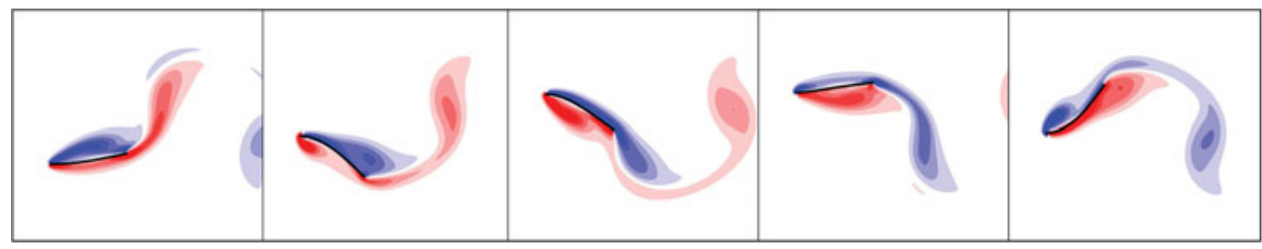

(c)

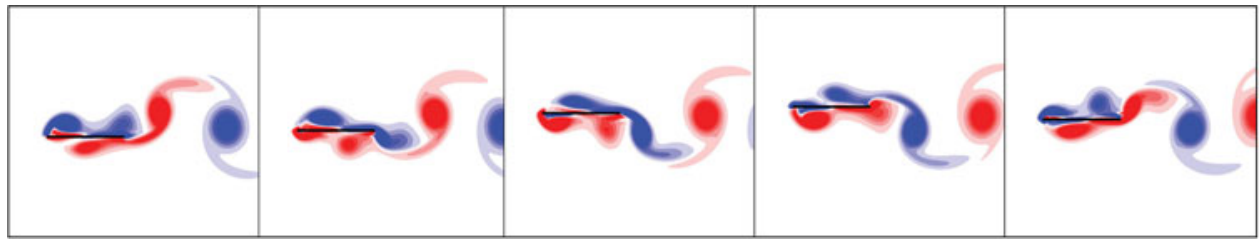

$(d)$

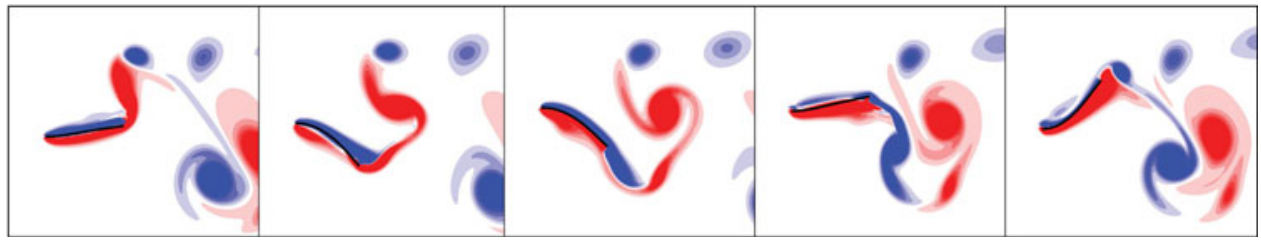

FIgURE 8. (Colour online) Vortex structures and foil shapes at five different moments within one flapping cycle: $(a)$ case $\mathrm{A} 1,(b)$ case $\mathrm{A} 2,(c)$ case $\mathrm{B} 1$ and $(d)$ case $\mathrm{B} 2$. Here $T=\pi \bar{A}$ is the dimensionless oscillation period.

flapping velocities are presented in figure 11. From this figure, it is evident that the magnitude of the trailing-edge flapping velocity becomes much larger when the flexibility of the foil is increased. For both plunging and pitching foils, a large trailing-edge flapping velocity is typically correlated with strong vorticity production (see, for example, Michelin \& Smith 2009 and Schnipper, Andersen \& Bohr 2009).

The root-mean-square (r.m.s.) values of the effective angles of attack and the trailing-edge flapping velocities in the four cases are summarised in table 4. The effective angle of attack in case A2 is reduced by $50 \%$ compared with case A1, whereas the trailing-edge flapping velocity is increased by a factor of 1.35 . The effective angle of attack in case B2 is reduced by $43 \%$ compared with case B1, whereas the trailing-edge flapping velocity is increased by a factor of 2.88 . The competition between the two opposing effects of flexibility eventually determines the overall trend of the modification of the vortex circulation. It can be concluded that the effect of decreasing the angle of attack is dominant in pair (A1, A2), whereas the effect of increasing the trailing-edge velocity is dominant in pair (B1, B2). From table 4, it is also evident that the amplitude ratio of the flexible foil is a close approximation of the trailing-edge velocity ratio between the flexible foil and the rigid foil. This finding can be easily understood, as the flapping period is the same 


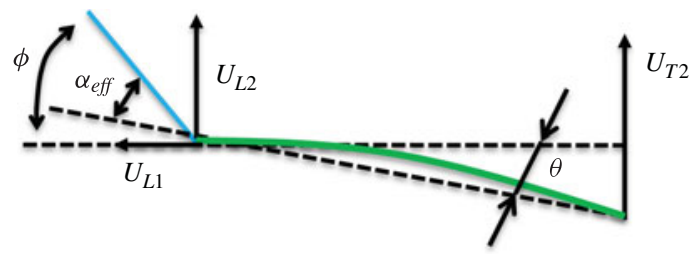

FIGURE 9. (Colour online) Definitions of the effective angle of attack, the angle of attack seen by a rigid foil and the deflection angle (the angle between the chord of the foil and the horizontal direction).
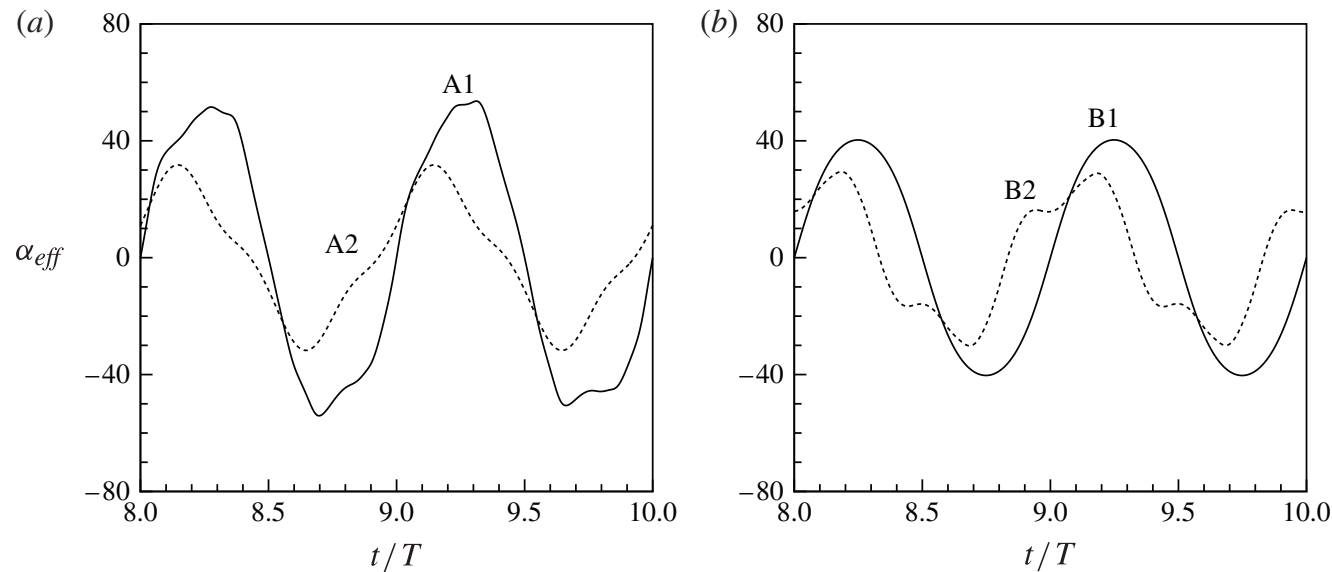

FIgURE 10. Time histories of the effective angles of attack for $(a)$ pair (A1, A2) and (b) pair (B1, B2). Here $T=\pi \bar{A}$ is the dimensionless oscillation period.
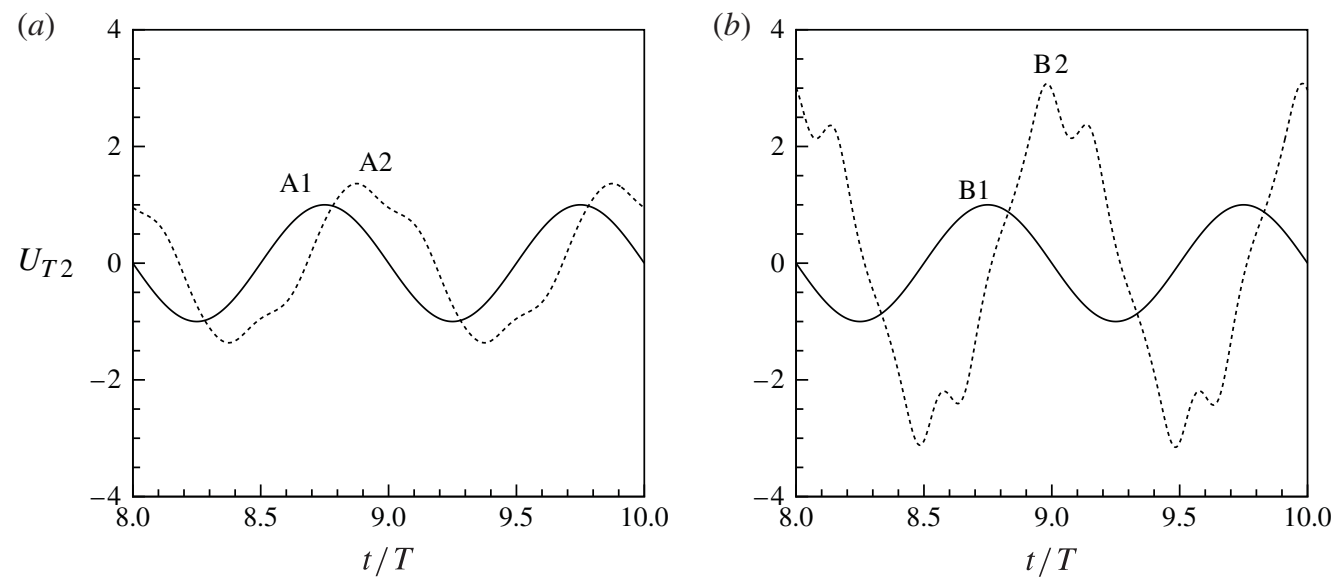

FIGURE 11. Time histories of the trailing-edge flapping velocities for (a) pair (A1, A2) and (b) pair (B1, B2). Here $T=\pi \bar{A}$ is the dimensionless oscillation period.

for both the flexible and rigid cases. It also explains why the amplitude ratio is the crucial parameter that dictates the symmetry properties of the wake. 


$\begin{array}{cccc} & \alpha_{\text {eff }}^{r m s}(\text { deg. }) & U_{T 2}^{r m s} & A_{\text {eff }} / A \\ \text { A1 } & 38.3 & 0.69 & 1.0 \\ \text { A2 } & 19.2 & 0.93 & 1.34 \\ \text { B1 } & 30.0 & 0.69 & 1.0 \\ \text { B2 } & 17.0 & 1.99 & 2.85\end{array}$

TABLE 4. Root-mean-square values of the effective angle of attack, r.m.s. values of the trailing-edge flapping velocity and amplitude-ratio values.

\subsection{Relation between structural resonance and wake symmetry}

It has been reported in some recent publications that harmonic (or superharmonic) structural resonance can be exploited to increase the transfer of energy to the fluid in flexible flapping-foil systems (Combes \& Daniel 2003; Michelin \& Smith 2009; Vanella et al. 2009; Masoud \& Alexeev 2010; Shyy et al. 2010; Kang et al. 2011); in other literature, the enhancement of propulsive efficiency has been attributed to the streamlined shape taken on by the foil as a result of deformation, rather than the effect of resonance (Thiria \& Godoy-Diana 2010; Ramananarivo et al. 2011). Thus, the exact role of resonance in flapping-based propulsion is still not fully understood. Here, in the context of these recent works, an endeavour is made to explore the relation between structural resonance and wake symmetry.

For this purpose, we analyse the results of two of the series of simulations listed in table 1 , namely, series $6(\bar{A}=1.0, \beta=0.2$, which includes case A1 and case A2) and series $12(\bar{A}=0.4, \quad \beta=2.0$, which includes case B1 and case B2). For each case in these two series, we evaluate the reduced forcing frequency, which is defined as the ratio of the forcing frequency $(f)$ to the first natural frequency $\left(f_{1}\right)$ of the filamentfluid system. The reduced forcing frequency increases with increasing flexibility, with a rigid limit of $f / f_{1}=0$ and a resonant point at $f / f_{1}=1$. For cases with large mass ratios (such as those in series 12), where the influence of the external fluid can be neglected, the $f_{1}$ value is approximately the same as that of a clamped-free elastic filament in vacuum. For the cases with small mass ratios (such as those in series 6), the natural frequencies of the system can be significantly affected by the presence of ambient fluid. Thus, we use the first natural frequency of a passive elastic filament in an axial flow (Michelin \& Smith 2009) as an approximation for $f_{1}$ in such cases.

The evolution of the vortex circulation, amplitude ratio and propulsive efficiency with increasing reduced forcing frequency is presented in figure 12. As discussed above, a wake transition (from a symmetric pattern to an asymmetric pattern or vice versa) occurs when the vortex circulation $\Gamma$ crosses a critical value. Because the LEV and the trailing-edge vortex (TEV) first amalgamate before they are shed into the wake as one single vortex, the vortex circulation depends on contributions from both the LEV and the TEV. The amplitude ratio is a natural indicator of structural resonance. From the discussion in $\S 3.3$, it is known that this parameter is also a good indicator of the TEV strength.

From the two efficiency curves presented in figure 12, it is evident that the optimal propulsive efficiency is achieved at a reduced forcing frequency that is much lower than the resonant point. More specifically, peak performance is achieved near $f / f_{1}=$ 0.7 for series 6 and near $f / f_{1}=0.4$ for series 12 . This finding is consistent with the observation reported by Ramananarivo et al. (2011). From the slopes of the efficiency curves at the point of wake transition, it is clear that the occurrence of symmetry 

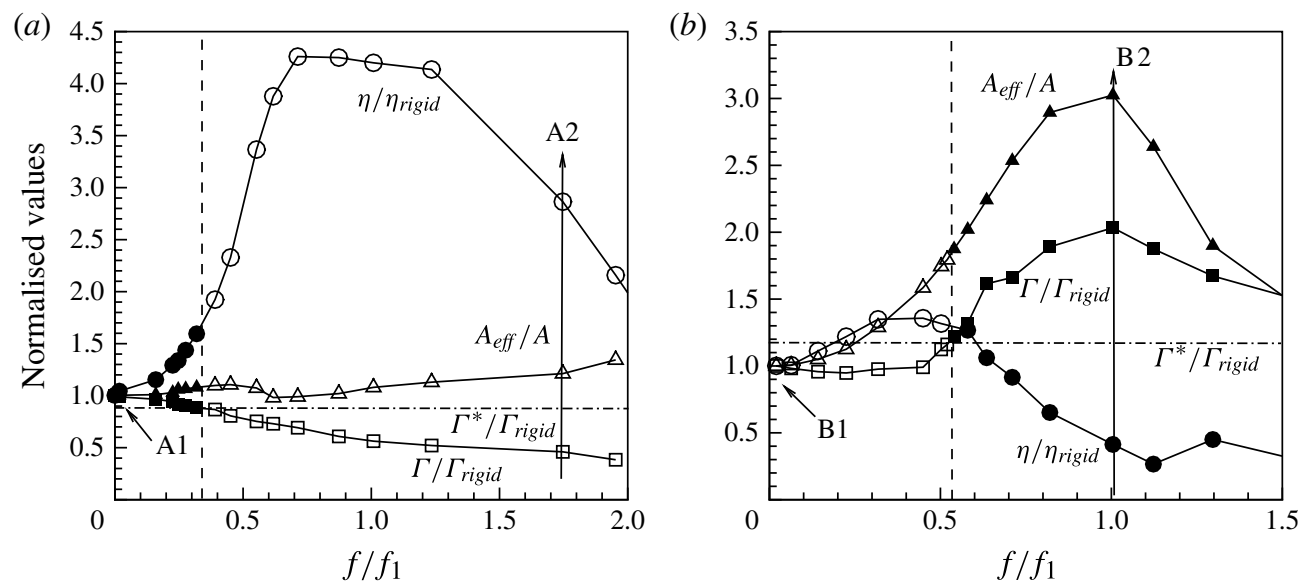

FIGURE 12. Normalised propulsive efficiency, normalised circulation and amplitude ratio as functions of the reduced forcing frequency for the cases considered in: $(a)$ series $6(\bar{A}=1.0 ; \beta=0.2)$; and $(b)$ series $12(\bar{A}=0.4 ; \beta=2.0)$. The circles and the solid lines connecting them represent propulsive efficiency. The squares and the solid lines connecting them represent vortex circulation. The triangles and the solid lines connecting them represent amplitude ratios. The propulsive efficiency and circulation are normalised by the values obtained in the rigid foil $\left(\gamma=10^{4}\right)$. The empty symbols represent cases with symmetric wakes, and the filled symbols represent cases with asymmetric wakes. The horizontal dash-dotted lines represent the critical values of the vortex circulation $\left(\Gamma^{*}\right)$ that corresponds to wake transitions. The vertical dashed lines represent the critical values of the reduced forcing frequency that correspond to wake transitions.

breaking leads to a decline in efficiency, as claimed in the introduction. From the two amplitude-ratio curves presented in figure 12, it is evident that the evolution of the amplitude ratio exhibits very different features for series 6 than for series 12 . The amplitude ratio achieved in series 6 is much lower than that in series 12 . For example, at the point of wake transition, the amplitude ratio is only 1.1 in series 6 , whereas it is approximately 1.9 in series 12 . In the amplitude-ratio curve for series 6 , a slight and rather broad peak is observed at $f / f_{1}=0.3-0.4$, indicating the occurrence of superharmonic resonance. Moreover, no clear resonance is observed near $f / f_{1}=1.0$. In a previous study by Ramananarivo et al. (2011), the superharmonic behaviour was associated with the nonlinearity in the structural equation, and the lack of a peak near $f / f_{1}=1.0$ was attributed to the effect of nonlinear fluid damping. In the amplituderatio curve for series 12 , however, no superharmonic behaviour is observed, but clear resonance is evident at $f / f_{1}=1.0$. These two different features observed in series 12 compared with series 6 can be understood as follows: $(a)$ the nonlinearity in the structural equation is weakened by a reduced flapping amplitude; and $(b)$ the effect of fluid damping is also weakened because the fluid force becomes less important at larger mass ratios.

From figure 12, it is apparent that the wake transitions occur at a reduced forcing frequency much lower than the resonant point (at $f / f_{1}=0.32$ for series 6 and $f / f_{1}=0.52$ for series 12). At first glance, it would seem that the wake symmetry properties and the structural resonance are completely unrelated to each other. However, after a more in-depth analysis, a more profound understanding can be attained. By comparing the trend in the vortex circulation with that in the amplitude 
ratio, we find that for series 6 and series 12, the relation between the wake symmetry properties and the structural resonance are very different in nature. For series 6, the vortex circulation $\Gamma$ decreases with increasing reduced forcing frequency. This decrease in $\Gamma$ is attributable to the reduced LEV strength, which is caused by the suppression of flow separation. Although the TEV strength increases marginally because of the slight increase in trailing-edge amplitude and velocity, its effect on the vortex circulation is completely overwhelmed by that of the attenuated LEV. The wake transition eventually occurs when the vortex circulation falls below the critical value. Thus, we can conclude that for series 6 , the wake transition cannot be associated with the resonance effect. For series 12, a plateau in the vortex circulation is initially observed at $0<f / f_{1}<0.45$ as the reduced forcing frequency is increased (from the rigid limit). The minimal variation in circulation in this range is attributed to the cancellation between an attenuated LEV and an intensified TEV. As the reduced forcing frequency is increased beyond $f / f_{1}=0.45$, however, the effect of the intensified TEV becomes the dominant factor influencing the vortex circulation. The trends observed in the vortex circulation and the trailing-edge amplitude with this increase in reduced forcing frequency are strongly correlated with each other, and the maxima of both quantities are also achieved at the same reduced forcing frequency, i.e. the resonant point. The wake transition occurs when the vortex circulation exceeds the critical value. Although this transition occurs at a reduced forcing frequency much lower than the resonant point, a close relation between the wake transition and the structural resonance is evident from the observations.

In the recent works cited above, two mechanisms that dictate the effects of flexibility on flapping-foil systems have been proposed, namely, the purely hydrodynamic effect attributable to deformation and the occurrence of structural resonance. By exploring a wide range of parameters, we observed the manifestation of both mechanisms in the present study. Based on the analysis presented above, we find that the wake transition near the lower transitional border depicted in figure $3(d)$ (where flexibility inhibits symmetry breaking) is associated with the purely hydrodynamic effect, whereas the wake transition near the upper transitional border depicted in figure $3(d)$ (where flexibility triggers symmetry breaking) is associated with the resonance effect.

\section{Conclusions}

We numerically studied the effects of the foil flexibility on the symmetry properties of the wake behind a self-propelled plunging foil. Our results indicate that the wake can either be stabilised or destabilised (in the sense of symmetry preservation) by increased flexibility. We demonstrated that for symmetry breaking to occur, the (dimensionless) vortex circulation must exceed a certain threshold value. The modification of vortex circulation is found to be the pivotal factor in the influence of the foil flexibility on the wake symmetry properties. Decreasing vortex circulation tends to stabilise the wake while increasing vortex circulation tends to destabilise it.

Increasing the flexibility of the foil can exert two opposing effects on the strength of vortex circulation. On one hand, increased flexibility reduces vorticity production near the leading edge by causing the foil to take on a 'streamlined' shape (with a reduced effective angle of attack); on the other hand, flexibility also enhances local vorticity production by increasing the trailing-edge flapping velocity. If the former effect is dominant, the wake is stabilised (symmetry breaking is inhibited). Conversely, if the latter effect is dominant, the wake is destabilised (symmetry breaking is triggered). 
Further investigation indicates that the former effect is purely hydrodynamic, whereas the latter effect can be associated with the structural resonance.

Finally, it is necessary to note that wake symmetry properties are closely related to efficiency and maneuverability. Symmetry breaking must be avoided during cruising, as propulsive efficiency is reduced if an asymmetric wake arises. However, symmetry breaking can be exploited for manoeuvring, as an asymmetric wake is automatically accompanied by a net lift and torque. In light of these considerations, flexibility can be exploited by animals to achieve high efficiency or to perform manoeuvres. The results of this work provide new insights into the functional role of passive flexibility in flapping-based biolocomotion.

\section{Acknowledgements}

This work was supported by the National Natural Science Foundation of China (grant numbers 11021262, 11023001, 11232011 and 11372331) and the National Basic Research Program of China (grant number 2013CB834100). We are grateful to Professor Z. C. Zheng for valuable discussions regarding the computation of vortex circulation.

\section{REFERENCES}

Alben, S., Witt, C., Baker, T. V., Anderson, E. \& Lauder, G. V. 2012 Locomotion of a passively flapping flat plate. Phys. Fluids 24, 051901.

Anderson, J. M., Streitlien, K., Barret, D. S. \& Triantafyllou, M. S. 1998 Oscillating foils for high propulsive efficiency. J. Fluid Mech. 360, 46-72.

BoraZjAni, I. \& Sotiropoulos, F. 2008 Numerical investigation of the hydrodynamics of carangiform swimming in the transitional and inertial flow regimes. J. Expl Biol. 211, $1541-1558$.

Cleaver, D. J., Wang, Z. \& Gursul, I. 2012 Bifurcating flows of plunging aerofoils at high Strouhal numbers. J. Fluid Mech. 708, 349-376.

Combes, S. A. \& Daniel, J. 2003 Flexural stiffness in insect wings II. Spatial distribution and dynamic wing bending. J. Expl Biol. 206, 2989-2997.

Eldredge, J. D., Toomey, J. \& Medina, A. 2010 On the roles of chord-wise flexibility in a flapping wing with hovering kinematics. J. Fluid Mech. 659, 94-115.

Godoy-Diana, R., Aider, J. L. \& Wesfreid, J. E. 2008 Transition in the wake of a flapping foil. Phys. Rev. E 77, 016308.

Godoy-Diana, R., Marais, C., Aider, J. L. \& Wesfreid, J. E. 2009 A model for the symmetry breaking of the reverse Benard-von Karman vortex street produced by a flapping foil. J. Fluid Mech. 622, 23-32.

HeAthCote, S. \& GURSUl, I. 2007a Flexible flapping airfoil propulsion at low Reynolds numbers. AIAA J. 45, 1066-1079.

HeAthCote, S. \& GURSUL, I. $2007 b$ Jet switching phenomenon for a periodically plunging airfoil. Phys. Fluids 19, 027104.

Huang, W. X., Shin, S. J. \& Sung, H. J. 2007 Simulation of flexible filaments in a uniform flow by the immersed boundary method. J. Comput. Phys. 226, 2206-2228.

Jones, K. D., Dohring, C. M. \& Platzer, M. F. 1998 Experimental and computational investigation of the Knoller-Betz effect. AIAA J. 36, 1240-1246.

Kang, C. K., Aono, H., Cesnik, C. E. S. \& Shyly, W. 2011 Effects of flexibility on the aerodynamic performance of flapping wings. J. Fluid Mech. 689, 32-74.

KATZ, J. \& WEIHS, D. 1978 Hydrodynamic propulsion by large amplitude oscillation of an airfoil with chordwise flexibility. J. Fluid Mech. 88, 713-723.

Kern, S. \& Koumoutsakos, P. 2006 Simulations of optimized anguilliform swimming. J. Expl Biol. 209, 4841-4857. 
Lauder, G. V., Anderson, E. J., Tangorra, J. \& Madden, P. G. 2007 Fish biorobotics: kinematics and hydrodynamics of self-propulsion. J. Expl Biol. 210, 2767-2780.

LEE, J. \& LEe, S. 2013 Fluid-structure interaction for the propulsive velocity of a flapping flexible plate at low Reynolds number. Comput. Fluids 71, 348-374.

Liang, C. L., Ou, K., Premasuthan, S., Jameson, A. \& Wang, Z. J. 2011 High-order accurate simulations of unsteady flow past plunging and pitching airfoils. Comput. Fluids 40, 236-248.

Marais, C., Thiria, B., Wesfreid, J. E. \& Godoy-Diana, R. 2012 Stabilizing effect of flexibility in the wake of a flapping foil. J. Fluid Mech. 710, 659-669.

Masoud, H. \& AleXeev, A. 2010 Resonance of flexible flapping wings at low Reynolds number. Phys. Rev. E 81, 056304.

Michelin, S.\& SMith, S. G. L. 2009 Resonance and propulsion performance of a heaving flexible wing. Phys. Fluids 21, 071902.

Prempraneech, P., Hover, F. S. \& Triantafyllou, M. S. 2003 The effect of chordwise flexibility on the thrust and efficiency of a flapping foil. In Proceedings of 13th International Symposium on Unmanned Untethered Submersible Technology, UUST, Durham, NH, USA.

Ramananarivo, S., Godoy-Diana, R. \& ThiRia, B. 2011 Rather than resonance, flapping wing flyers may play on aerodynamics to improve performance. Proc. Natl Acad. Sci. USA 108, 5964-5969.

Schnipper, T., Andersen, A. \& Bohr, T. 2009 Vortex wakes of a flapping foil. J. Fluid Mech. 633, 411-423.

Schultz, W. W. \& WebB, P. W. 2002 Power requirements of swimming: do new methods resolve old questions? Integr. Compar. Biol. 42, 1018-1025.

Shoele, K. \& ZHU, Q. 2012 Leading edge strengthening and the propulsion performance of flexible ray fins. J. Fluid Mech. 693, 402-432.

Shyy, W., Aono, H., Chimakurthi, S. K., Trizila, P., Kang, C. K., Cesnik, C. E. S. \& LIU, H. 2010 Recent progress in flapping wing aerodynamics and aeroelasticity. Prog. Aerosp. Sci. 46, 284-327.

Spagnolie, S. E., Moret, L., Shelley, M. J. \& Zhang, J. 2010 Surprising behaviours in flapping locomotion with passive pitching. Phys. Fluids 22, 041903.

Thiria, B. \& Godoy-DianA, R. 2010 How wing compliance drives the efficiency of self-propelled flapping flyers. Phys. Rev. E 82, 015303(R).

Vanella, M., Fitzgerald, T., Preidikman, S., Balaras, E. \& Balachandran, B. 2009 Influence of flexibility on the aerodynamic performance of a hovering wing. J. Expl Biol. 212, 95-105.

WANG, S. Z.\& ZHANG, X. 2011 An immersed boundary method based on discrete stream function formulation for two- and three-dimensional incompressible flows. J. Comput. Phys. 230, 3479-3499.

Zhang, J., LiU, N. S. \& LU, X. Y. 2010 Locomotion of a passively flapping flat plate. J. Fluid Mech. 659, 43-68.

Zheng, Z. C. \& WeI, Z. 2012 Study of mechanisms and factors that influence the formation of vortical wake of a heaving airfoil. Phys. Fluids 24, 103601.

ZHU, X. J., HE, G. W. \& ZHANG, X. 2014 An improved direct-forcing immersed boundary method for fluid-structure interaction simulations. Trans. ASME J. Fluids Engng 136, 040903. 